\title{
Fast-Diffusion Limit with Large Noise for Systems of Stochastic Reaction-Diffusion Equations
}

\author{
Wael W. Mohammed* Dirk Blömker ${ }^{\dagger}$
}

May 30, 2016

\begin{abstract}
We consider a class of a stochastic reaction-diffusion equations with additive noise. In the limit of fast diffusion one can approximate solutions of the stochastic reaction-diffusion equations by the solution of a suitable system of ordinary differential equation only describing the reactions, but due to nonlinear interaction of large diffusion and fluctuations in the limit new effective reaction terms appear.

We focus on systems with polynomial nonlinearities and illustrate the result by applying it to a predator-prey system and a cubic autocatalytic reaction between two chemicals.
\end{abstract}

Keywords: Reaction Diffusion System, Averaging, Fast Diffusion Limit

\section{Introduction}

Reaction diffusion systems are mathematical models that describe how the concentration of one or more substances distributed in space changes. The main features for the dynamics are on one hand the influence of local chemical reactions, in which the substances are converted into each other, and on the other hand diffusion, which causes the substances to spread out in space. In the special case of all reactions being on the same time-scale, we consider here the case of fast diffusion in nonlinear interaction with large mass-conservative noise, which in the limit may lead to new effective reaction terms, although the noise itself is not present in the limiting equation. The also interesting case of a few diffusions being fast, with a limiting system of stochastic ordinary and partial equations is not studied here.

\footnotetext{
*Department of Mathematics, Faculty of Science, Mansoura University, Egypt, wael.mohammed@mans.edu.eg

${ }^{\dagger}$ Institut für Mathematik, Universität Augsburg, Germany, dirk.bloemker@math.uniaugsburg. de
} 
Reaction-diffusion equations are not limited to the field of chemistry and chemical engineering. They can describe the dynamics of non-chemical systems, and reaction-diffusion equations provide a general theoretical framework for the study of phenomena in areas such as biology, ecology, physics, and materials science. We give two probably oversimplified and not realistic examples to illustrate our results in applications.

Let us remark that S. Cerrai $[7,8,9]$ and S. Cerrai and M. Freidlin [10] have studied the validity of an averaging principle and the deviations of the dynamics from the averaged motion of systems of stochastic reactiondiffusion type perturbed by a noise of multiplicative type.

In this article we consider the following system of stochastic reactiondiffusion equations for $n$ species with respect to no flux Neumann boundary conditions on a bounded smooth domain $G$ :

$$
\frac{\partial u_{i}}{\partial t}=\frac{d_{i}}{\varepsilon^{2}} \Delta u_{i}+\mathcal{F}_{i}\left(u_{1}, u_{2}, \ldots ., u_{n}\right)+\sigma_{\varepsilon} \partial_{t} W_{i}(t), \quad \text { for } i=1,2, . ., n,
$$

where $\mathcal{F}_{i}\left(u_{1}, u_{2}, \ldots . ., u_{n}\right)$ are polynomials of degree $m_{i}$ (i.e. linear combinations of monomials $u_{1}^{\ell_{1}} \cdot u_{n}^{\ell_{n}}$ with $\sum_{j=1}^{n} \ell_{j} \leq m_{i}$ ) and the stochastic forcing $\left\{W_{i}(t)\right\}_{t \geq 0}$ are finite dimensional Wiener processes. Our aim is to establish rigorously error estimates for the fast-diffusion limit $\varepsilon \rightarrow 0$ for (1).

We do not study the details on the existence and uniqueness of solutions here, which is a well settled problem. We always assume that there is at least a unique local solution. See for example F. Flandoli [16] for the global existence and uniqueness for a stochastic reaction-diffusion equation with polynomial nonlinearity in a bounded domain.

In this paper, we study only cases where the noise is not changing the overall average and is large, i.e $\sigma_{\varepsilon}=\varepsilon^{-1}$. We comment on other possible noise terms further below.

In this setting, our main results verify that the solutions of Equation (1) are well approximated by

$$
u_{i}(t, x)=b_{i}(t)+\mathcal{Z}_{i}(t, x)+\text { error },
$$

where $b$ represents the average concentration of the components of $u$. The stochastic process $\mathcal{Z}_{i}(t, x)$, defined later in (6), is an $\varepsilon$-dependent fast OrnsteinUhlenbeck corresponding to noise in the limit $\varepsilon \rightarrow 0$. But it does not effect the average concentration directly. The error term has an explicit bound on arbitrary moments of it in the sense of Definition 7 .

Our main result (Theorem 9) shows that $b$ is given by the following system of ordinary differential equations

$$
\partial_{t} b_{i}(t)=\overline{\mathcal{F}}_{i}(b), \text { for } i=1,2, . ., n,
$$

with an averaged non-linearity

$$
\overline{\mathcal{F}}_{i}(b)=\int_{\mathcal{H}^{\alpha}} \mathcal{F}_{i}(b+z) d \mu(z),
$$


where $\mu$ is the $\varepsilon$-independent invariant measure of the fast Ornstein-Uhlenbeck process $\mathcal{Z}$. Observe that the $\varepsilon$-dependence of $\mathcal{Z}$ arises mainly by considering it on a slower time-scale, which does not lead to an $\varepsilon$-dependence of the invariant measure $\mu$.

Results of these types without error estimates are well-known in averaging theory, see for instance $[21,22,27]$ and the reference therein. One main novelty of our approach here and in other publications is the explicit error estimate in terms of arbitrarily high moments of the error, while usually only weak convergence towards the approximation is treated.

Equation (2) is a system of ordinary differential equations and it describes the reaction without diffusion. The equation is without noise, as we consider degenerate noise that has no direct impact on the mean average $b$. Also due to the nature of the equation no multiplicative noise term can appear as for example in the work on [20] on amplitude equations, which uses a related approach.

It is a somewhat surprising result that in case $\overline{\mathcal{F}}_{i} \neq \mathcal{F}_{i}$ large noise due to its nonlinear interaction with diffusion has the potential to generate new effective reaction terms. This is an effect known from SDEs like the Duffing van der Pole oscillator (cf. [15], see also [6]). It was observed in scalar SPDEs, too. See for example $[18,19,25]$ and for rigorous error estimates also $[4,5,23]$. In these examples due to noise and nonlinear interaction an additional linear term was observed in the averaged equation, which yields a change in the point of bifurcation. Here in the reaction system under consideration all kinds of different additional reaction terms may appear.

Let us finally remark on the nature of our noise. First, if we consider noise of smaller intensity, then completely analogous results, to the ones presented here, hold. Only in the final result the whole impact of the noise is lost, and the limiting system is just the deterministic system $\partial_{t} b=\mathcal{F}(b)$ without additional terms. Moreover, too large noise will destroy the result, and only noise is observed for $b$. So $\sigma_{\varepsilon}=\varepsilon^{-1}$ is the intermediate regime, where both the impact of noise and the nonlinear effect from the reaction sustain in the limiting equation for $b$.

Secondly, we are interested on mass-conservative noise that directly cannot have an impact on the average. If we consider noise with direct impact on the average concentration, then the correct scaling is $\sigma_{\varepsilon}=1$. In this case the limiting equation should be $\partial_{t} b=\mathcal{F}(b)$ plus an additional noise term, arising from projection of the noise onto the constant functions. This could be established with the strategy of proof presented in $[1,3]$ without any further technical difficulties. Moreover, as the result is the one that is expected, we do not treat this case here.

Our general strategy of proof is based on [4] or [23] and we use technical lemmas established in our work there, but systems of SPDEs were not in the scope of the results in [4] or [23]. Moreover, as the problem here is posed directly on the fast time-scale many additional interaction between 
noise and nonlinearity are possible, and in the averaged equation all kinds of effective reaction terms are induced by the noise.

The remainder of the paper is organized as follows. In Section 2 we state the precise setting for equation (1) and the assumptions that we need. In Section 3 we derive the fast-diffusion limit with explicit error estimates and present the main theorem of this paper. In Section 4 we give the proof of the main result, while in Section 5 we give an averaging lemma with error bounds, which is used in the proof of the main result, and derive an explicit formula for $\overline{\mathcal{F}}_{i}$. Finally, we give two probably oversimplified examples from biology and chemistry, in order to illustrate our results, which can be applied to many phenomena in different areas.

\section{Definition and Assumptions}

This section states the precise setting for the formal SPDE (1) and summarizes all assumptions necessary for our results. For simplicity in the analysis we work in the separable Hilbert space $\mathcal{H}=\mathcal{L}^{2}(G)$, where $G \subset \mathbb{R}^{d}$ is a bounded domain and $\partial G$ is its sufficiently smooth boundary (e.g. Lipschitz), equipped with scalar product $\langle\cdot, \cdot\rangle$ and norm $\|\cdot\|$.

Definition 1 For $i=1,2, \ldots, n$ and diffusion constants $d_{i}>0$ define

$$
\mathcal{A}_{i}=d_{i} \Delta
$$

with

$$
D\left(\mathcal{A}_{i}\right)=\left\{u \in \mathcal{H}^{2}:\left.\partial_{\nu} u\right|_{\partial G}=0\right\},
$$

where $\partial_{\nu} u$ is the normal derivative of $u$ on $\partial G$.

It is well known that $\mathcal{A}_{i}$ has an orthonormal base of eigenfunctions $\left\{e_{k}\right\}_{k=0}^{\infty}$. For all $i$ it is the same basis $e_{k}$ in $\mathcal{H}$ with corresponding eigenvalues $\left\{d_{i} \lambda_{k}\right\}_{k=0}^{\infty}$ depending on $i$ (cf. Courant and Hilbert [11]).

The kernel of $\mathcal{A}_{i}$ is given by the constants, i.e.

$$
\mathcal{N}:=\operatorname{ker} \mathcal{A}_{i}=\operatorname{span}\left\{e_{0}\right\},
$$

where $e_{0}$ is spatially constant with corresponding eigenvalue $\lambda_{0}=0$. Define the orthogonal complement $S=\mathcal{N}^{\perp}$ of $\mathcal{N}$ in $\mathcal{H}$, and the orthogonal projection $P_{c} u=\frac{1}{|G|} \int_{G} u d x$ (for all $u \in \mathcal{H}$ ) onto $\mathcal{N}$. Moreover, define the projection $P_{s} u:=\left(\mathcal{I}-P_{c}\right) u$ onto the orthogonal complement $S$, where $\mathcal{I}$ is the identity operator on $\mathcal{H}$.

With a slight abuse of notation, we say $a \in \mathcal{N}$ for a vector valued $a \in\left(\mathcal{H}^{\alpha}\right)^{n}$, if for all components $a_{i} \in \mathcal{N}$. Similarly, $\psi \in \mathcal{S}$ for $\psi \in\left(\mathcal{H}^{\alpha}\right)^{n}$, if for all components $\psi_{i} \in \mathcal{S}$. 
Definition 2 For $\alpha>\frac{1}{2}$ we define the fractional interpolation space $\mathcal{H}^{\alpha}$ as $\mathcal{H}^{\alpha}=\left\{\sum_{k=0}^{\infty} \gamma_{k} e_{k}: \sum_{k=1}^{\infty} \gamma_{k}^{2} k^{2 \alpha}<\infty\right\}$ with norm $\left\|\sum_{k=0}^{\infty} \gamma_{k} e_{k}\right\|_{\alpha}^{2}=\gamma_{0}^{2}+\sum_{k=1}^{\infty} \gamma_{k}^{2} k^{2 \alpha}$.

Note that $\lambda_{k} \sim k^{2 / d}$ for the eigenvalues of the Laplacian in $G \subset \mathbb{R}^{d}$, and thus our space $\mathcal{H}^{\alpha}$ corresponds to the usual Sobolev space $H^{d \alpha}(G)$. Thus $\alpha>\frac{1}{2}$ implies that $\mathcal{H}^{\alpha}$ is embedded into $L^{\infty}(G)$. Moreover, one has $\|u v\|_{\alpha} \leq C\|u\|_{\alpha}\|u\|_{\alpha}$ for all $u, v \in \mathcal{H}^{\alpha}$ (cf. [26]).

The operator $\mathcal{A}_{i}$ given by Definition 1 generates an analytic semigroup $\left\{e^{t \mathcal{A}_{i}}\right\}_{t \geq 0}$ (cf. Dan Henry [17] or Pazy [28]), on any space $\mathcal{H}^{\alpha}$. This semigroup is defined by

$$
e^{\mathcal{A}_{i} t}\left(\sum_{k=1}^{\infty} \gamma_{k} e_{k}\right)=\sum_{k=1}^{\infty} e^{-d_{i} \lambda_{k} t} \gamma_{k} e_{k} \quad \forall t \geq 0 .
$$

It is well known, that there is an $\omega>0$ such that for all $t>0, i=1,2, \ldots . n$ and all $u \in \mathcal{H}^{\alpha}$

$$
\left\|e^{t \mathcal{A}_{i}} P_{s} u\right\|_{\alpha} \leq e^{-\omega t}\left\|P_{s} u\right\|_{\alpha}
$$

where $\omega$ depends in general on $d_{i}$.

For the noise we suppose that it is finite dimensional and thus solutions are always spatially smooth:

Assumption 3 For $i=1,2, \ldots . ., n$, let $W_{i}$ be a finite dimensional Wiener process on $\mathcal{H}$. Suppose for $t \geq 0$,

$$
W_{i}(t)=\sum_{k=1}^{N} \rho_{i k} \beta_{i k}(t) e_{k}, \text { for } i=1,2, . ., n,
$$

where the $\beta_{i k}$ are $\mathbb{R}$-valued standard Brownian motions and the $\rho_{i k}$ are real numbers.

Note that we do not assume for the general result that the $W_{i}$ are independent or the $\beta_{i k}$ are independent, but for the examples and the verification of the averaging in Assumption 5 later, for simplicity of calculation we will always assume that they all are independent. Let us remark that this assumption of independence although simplifying the final result substantially is quite restrictive, as the spatial correlation of the noise is quite limited. See [2] for a detailed discussion.

Definition 4 The fast Ornstein-Uhlenbeck process (OU-process, for short) $\mathcal{Z}_{i}$ is defined as

$$
\mathcal{Z}_{i}(t)=\sum_{k=1}^{N} \mathcal{Z}_{i k} e_{k}
$$


with

$$
\mathcal{Z}_{i k}(t)=\rho_{i k} \varepsilon^{-1} \int_{0}^{t} e^{-\varepsilon^{-2}(t-\tau) d_{i} \lambda_{k}} d \beta_{i k}(\tau) .
$$

For the polynomial $\mathcal{F}_{i}(u)$, we assume that it is a polynomial, and that an averaging property with respect to the OU-process holds.

Assumption 5 The nonlinearity $\mathcal{F}_{i}$ is for $i=1,2, . ., n$, a polynomial of degree $m_{i}$, and define $m$ as $m=\max \left(m_{1}, \ldots ., m_{n}\right)$.

Suppose furthermore that there is a polynomial $\overline{\mathcal{F}}_{i}$ of degree less than or equal $m_{i}$ such that for some small $\kappa>0$ and all $p>1$ there is a constant $C>0$ such that for all adapted stochastic processes with $(d u)^{2}=0$ and $\tau^{*}=\inf \left\{t>0:\|u(t)\|_{\alpha}>\varepsilon^{-\kappa}\right\}$

$$
\mathbb{E} \sup _{\left[0, \tau^{*}\right]}\left\|\mathcal{F}_{i}\left(u+\mathcal{Z}_{i}\right)-\overline{\mathcal{F}}_{i}(u)\right\|_{\alpha}^{p} \leq C \varepsilon^{p-p m_{i} \kappa}
$$

The second half of the assumption is an averaging result that will be studied in detail in Section 5 .

Let us state a brute force estimate based on Assumption 5. For our polynomials as $\alpha>\frac{1}{2}$ we can always bounded them by the highest power and a constant.

$$
\left\|\mathcal{F}_{i}(u)\right\|_{\alpha} \leq C\left(1+\|u\|_{\alpha}^{m_{i}}\right) \text { for all } u \in\left(\mathcal{H}^{\alpha}\right)^{n} .
$$

Let us remark that under Assumption 5 the nonlinearity $\mathcal{F}$ is a locally Lipschitz-continuous map from $\left(\mathcal{H}^{\alpha}\right)^{n}$ into $\left(\mathcal{H}^{\alpha}\right)^{n}$. Thus it is standard (cf. Da Prato and Zabczyk [12]) to verify that there is a unique local $\left(\mathcal{H}^{\alpha}\right)^{n}$ valued solution $u$ that is continuous and exists until one of its components blows up.

For our approximation result we rely on a cut off argument. We consider only solutions that are not too large, as given by the next definition.

Definition 6 For the $\left(\mathcal{H}^{\alpha}\right)^{n}$-valued solution u defined later in (11), we define for some $T_{0}>0$ and small $\kappa \in\left(0, \frac{1}{2 m+1}\right)$ the stopping time $\tau^{*}$ as

$$
\tau^{*}:=T_{0} \wedge \inf \left\{t>0:\|u(t)\|_{\alpha}>\varepsilon^{-\kappa}\right\} .
$$

Here $\wedge$ denotes the minimum of the two terms.

We give error estimates in terms of the following $\mathcal{O}$-notation.

Definition 7 For a real-valued family of processes $\left\{X_{\varepsilon}(t)\right\}_{t \geq 0}$ we say that $X_{\varepsilon}$ is of order $f_{\varepsilon}$, i.e. $X_{\varepsilon}=\mathcal{O}\left(f_{\varepsilon}\right)$, if for every $p \geq 1$ there exists a constant $C_{p}$ such that

$$
\mathbb{E} \sup _{t \in\left[0, \tau^{*}\right]}\left|X_{\varepsilon}(t)\right|^{p} \leq C_{p} f_{\varepsilon}^{p} .
$$

We use also the analogous notation for time-independent random variables. 
Definition 8 (Multi-Index Notation) Let $\ell \in \mathbb{N}_{0}^{n}$, i.e. $\ell=\left(\ell_{1}, \ell_{2}, \ldots \ldots, \ell_{n}\right)$ be a vector of nonnegative integers, $u=\left(u_{1}, u_{2}, \ldots u_{n}\right)$. Then we define:

$$
\begin{aligned}
|\ell| & =\ell_{1}+\ell_{2}+\ldots \ldots+\ell_{n} & \ell ! & =\ell_{1} ! \ell_{2} ! \ldots \ldots \ell_{n} ! \\
u^{\ell} & =u_{1}^{\ell_{1}} u_{2}^{\ell_{2}} \ldots u_{n}^{\ell_{n}} & D^{\ell} & =\partial_{u_{1}}^{\ell_{1}} \partial_{u_{2}}^{\ell_{2}} \ldots . . \partial_{u_{n}}^{\ell_{n}}
\end{aligned}
$$

\section{The approximation Theorem}

Before stating the main result, let us first discuss the formal derivation of the limiting equation corresponding to (1). We split the solution $u$ into

$$
u_{i}(t, x)=a_{i}(t)+\psi_{i}(t, x), \quad \text { for } i=1,2, \ldots, n,
$$

with the vector of averages $a \in \mathcal{N}$ and the vector of remainders $\psi \in \mathcal{S}$. Plugging (11) into (1) and projecting everything onto $\mathcal{N}$ and $\mathcal{S}$ we obtain for $i=1,2, \ldots ., n$ first in $\mathcal{N}$

$$
\partial_{t} a_{i}=\mathcal{F}_{i}^{c}(a+\psi)
$$

and then in $\mathcal{S}$

$$
\partial_{t} \psi_{i}=\frac{1}{\varepsilon^{2}} \mathcal{A} \psi_{i}+\mathcal{F}_{i}^{s}(a+\psi)+\frac{1}{\varepsilon} \partial_{t} W_{i}(t)
$$

where we used the short-hand notation $\mathcal{F}_{i}^{c}=P_{c} \mathcal{F}_{i}$ and $\mathcal{F}_{i}^{s}=P_{s} \mathcal{F}_{i}$. Equations (12) is stated in the integrated form as

$$
a_{i}(t)=a_{i}(0)+\int_{0}^{t} \mathcal{F}_{i}^{c}(a(\tau)+\psi(\tau)) d \tau,
$$

and (13) in the mild formulation as

$$
\psi_{i}(t)=e^{\varepsilon^{-2} t \mathcal{A}} \psi_{i}(0)+\int_{0}^{t} e^{\varepsilon^{-2}(t-\tau) \mathcal{A}_{s}} \mathcal{F}_{i}^{s}(a(\tau)+\psi(\tau)) d \tau+\mathcal{Z}_{i}(t),
$$

for $i=1, . ., n$, where the fast OU-process $\mathcal{Z}_{i}$ was defined in Definition 4 . Formally, we see that in first approximation $\psi$ equals the OU-process $\mathcal{Z}$ (cf. Lemma 10 in next section for the rigorous approximation). Thus, we can replace $\psi$ by $\mathcal{Z}$ in Eq. (14), in order to obtain

$$
a_{i}(t) \approx a_{i}(0)+\int_{0}^{t} \mathcal{F}_{i}^{c}(a(\tau)+\mathcal{Z}(\tau)) d \tau .
$$

Using Assumption 5, yields the averaged equation with error term

$$
a_{i}(t)=a_{i}(0)+\int_{0}^{t} \overline{\mathcal{F}}_{i}(a(\tau)) d \tau+\mathcal{O}\left(\varepsilon^{1-2 m \kappa}\right) .
$$

Now the main result of this paper is: 
Theorem 9 (Approximation) Under Assumptions 3 and 5 let $u$ be a solution of (1) with splitting $u=a+\psi$ defined in (11) with the initial condition $u(0)=a(0)+\psi(0)$ with $a(0) \in \mathcal{N}$ and $\psi(0) \in S$ where $a(0)$ and $\psi(0)$ are of order one, and $b$ is a solution of (2) with $b(0)=a(0)$. Then for all $p>0, C_{0}>0$ and $T_{0} \geq T_{1}>0$ and all $\kappa \in\left(0, \frac{1}{2 m+1}\right)$, there exists $C>0$ such that

$$
\mathbb{P}\left(\sup _{t \in\left[0, T_{1}\right]}\|u(t)-b(t)-\mathcal{Q}(t)\|_{\alpha}>\varepsilon^{1-2 m \kappa-\kappa}\right) \leq C \varepsilon^{p}+\mathbb{P}\left(\sup _{\left[0, T_{1}\right]}\|b\|_{\alpha}>C_{0}\right),
$$

where

$$
\mathcal{Q}(t)=e^{\varepsilon^{-2} t \mathcal{A}_{s}} \psi(0)+\mathcal{Z}(t),
$$

with fast $O U$-process $\mathcal{Z}$ defined in (6).

Note that in the proof, we will fix $\kappa$, but we always think of it as being sufficiently small.

We see that the first part of (17) depending on the initial condition decays exponentially fast on the time-scale of order $\mathcal{O}\left(\varepsilon^{2}\right)$, while $\mathcal{Z}$ is a fast OU-process that approximates noise.

\section{Proof of the Main Result}

For the whole section, let $u$ be a solution of (1) with splitting $u=a+$ $\psi$ defined in (11). In the first lemma of this section, we see that $\psi_{i}$ is approximately equal to the fast Ornstein-Uhlenbeck process $\mathcal{Z}_{i}$ defined in (6).

Lemma 10 Under Assumption 5 there is a constant $C>0$ such that for $\kappa>0$ from the definition of $\tau^{*}$ and $p \geq 1$

$$
\mathbb{E} \sup _{t \in\left[0, \tau^{*}\right]}\left\|\psi_{i}(t)-e^{\varepsilon^{-2} t \mathcal{A}} \psi_{i}(0)-\mathcal{Z}_{i}(t)\right\|_{\alpha}^{p} \leq C \varepsilon^{2 p-m_{i} p \kappa},
$$

for $i=1,2, \ldots, n$, where $m_{i}$ is the degree of the polynomial $\mathcal{F}_{i}$.

Proof. From the mild formulation in (15) using triangle inequality, the semigroup estimate, and the fact that $\mathcal{F}_{i}$ is a polynomial of degree $m_{i}$, we obtain for $\alpha>\frac{1}{2}$

$$
\begin{aligned}
\left\|\psi_{i}(t)-\mathcal{Z}_{i}(t)-e^{\varepsilon^{-2} t \mathcal{A}} \psi_{i}(0)\right\|_{\alpha} & \leq\left\|\int_{0}^{t} e^{\varepsilon^{-2} \mathcal{A}_{s}(t-\tau)} \mathcal{F}_{i}^{s}(a+\psi) d \tau\right\|_{\alpha} \\
& \leq C \sup _{\tau \in\left[0, \tau^{*}\right]}\left\|\mathcal{F}_{i}^{s}(a+\psi)\right\|_{\alpha} \int_{0}^{t} e^{-\varepsilon^{-2} \omega(t-\tau)} d \tau \\
& \leq C \varepsilon^{2} \sup _{\tau \in\left[0, \tau^{*}\right]}\left(1+\|a+\psi\|_{\alpha}^{m_{i}}\right) \\
& \leq C \varepsilon^{2-m_{i} \kappa},
\end{aligned}
$$


where we used in the last inequality that by the definition of $\tau^{*}$ the solution $u$ and thus also both $a$ and $\psi$ are bounded by $C \varepsilon^{-\kappa}$.

We need later the following uniform bounds on the OU-process $\mathcal{Z}_{i k}(t)$ and $\mathcal{Z}_{i}(t)$ defined in $(7)$ and $(6)$, respectively.

Lemma 11 Under Assumption 3, for every $\kappa_{0}>0$ and $p \geq 1$ there is a constant $C$, depending on $p, \alpha_{k}, \lambda_{k}, \kappa_{0}$ and $T_{0}$, such that

$$
\mathbb{E} \sup _{t \in\left[0, T_{0}\right]}\left\|\mathcal{Z}_{i k}(t)\right\|_{\alpha}^{p} \leq C \varepsilon^{-\kappa_{0}},
$$

and

$$
\mathbb{E} \sup _{t \in\left[0, T_{0}\right]}\left\|\mathcal{Z}_{i}(t)\right\|_{\alpha}^{p} \leq C \varepsilon^{-\kappa_{0}} .
$$

Proof. See the proof of Lemma 4.2 in [4].

The following corollary states that $\psi(t)$ is with high probability much smaller than $\varepsilon^{-\kappa}$ as asserted by the Definition 6 for $t \leq \tau^{*}$. We show later $\tau^{*} \geq T_{0}$ with high probability (cf. proof of Theorem 9 ).

Corollary 12 Under the assumptions of Lemmas 10 and 11, if $\psi(0)=$ $\mathcal{O}(1)$, then for $p>0$ there exists a constant $C>0$ such that

$$
\mathbb{E} \sup _{t \in\left[0, \tau^{*}\right]}\left\|\psi_{i}(t)\right\|_{\alpha}^{p} \leq C \varepsilon^{-\kappa_{0}}
$$

Proof. By triangle inequality and Lemma 11, we obtain from (18) for any fixed $p>1$ that

$$
\mathbb{E} \sup _{t \in\left[0, \tau^{*}\right]}\left\|\psi_{i}(t)\right\|_{\alpha}^{p} \leq C+C \varepsilon^{2 p-m_{i} p \kappa}+C \varepsilon^{-\kappa_{0}},
$$

which implies (21) provided $\kappa \leq \frac{2}{m_{i}}+\frac{\kappa_{0}}{m_{i} p}$.

Let us now state a result similar to averaging, in the case when we integrate over the fast decaying contribution of the initial condition in $\psi_{i}$. This leads to terms of order $\mathcal{O}\left(\varepsilon^{2}\right)$.

Lemma 13 For $q \geq 1$ there exists a constant $C>0$ such that

$$
\int_{0}^{T}\left\|e^{\tau \varepsilon^{-2} \mathcal{A}_{s}} \psi_{0}\right\|_{\alpha}^{q} d \tau \leq C \varepsilon^{2}\left\|\psi_{0}\right\|_{\alpha}^{q} \quad \text { for all } \psi_{0} \in \mathcal{H}^{\alpha} .
$$

Proof. Using (4) we obtain

$$
\int_{0}^{T}\left\|e^{\varepsilon^{-2} \mathcal{A}_{s} \tau} \psi_{0}\right\|_{\alpha}^{q} d \tau \leq c \int_{0}^{T} e^{-q \varepsilon^{-2} \omega \tau}\left\|\psi_{0}\right\|_{\alpha}^{q} d \tau \leq \frac{\varepsilon^{2}}{q \omega}\left\|\psi_{0}\right\|_{\alpha}^{q} .
$$


Lemma 14 Let Assumptions 3 and 5 hold. Then

$$
a_{i}(t)=a_{i}(0)+\int_{0}^{t} \overline{\mathcal{F}}_{i}(a) d \tau+\tilde{R}(t)
$$

with

$$
\tilde{R}=\mathcal{O}\left(\varepsilon^{1-2 m \kappa-\kappa_{0}}\right) .
$$

Proof. From the mild formulation in (15) and Lemma 10, we obtain

$$
\psi(t)=\mathcal{Z}(t)+e^{\varepsilon^{-2} t \mathcal{A}} \psi(0)+\mathcal{O}\left(\varepsilon^{2-m \kappa}\right) .
$$

Define

$$
y(t)=e^{\varepsilon^{-2} t \mathcal{A}} \psi(0) \quad \text { and } \quad R(t)=\mathcal{O}\left(\varepsilon^{2-m \kappa}\right) .
$$

Substituting from (24) into (14), yields

$$
a_{i}(t)=a_{i}(0)+\int_{0}^{t} \mathcal{F}_{i}^{c}(a+\mathcal{Z}+y+R)(\tau) d \tau .
$$

Applying Taylor's expansion to the polynomial $\mathcal{F}_{i}^{c}: \mathcal{H} \rightarrow \mathbb{R}$, yields

$$
a_{i}(t)=a_{i}(0)+\int_{0}^{t} \mathcal{F}_{i}^{c}(a+\mathcal{Z})(\tau) d \tau+R_{1}(t)
$$

where $R_{1}(t)$ is given by

$$
R_{1}(t)=\sum_{|\ell| \geq 1} P_{c} \int_{0}^{t} \frac{D^{\ell} \mathcal{F}_{i}(a+\mathcal{Z})}{\ell !}(y+R)^{\ell} d \tau,
$$

and the sum is finite as $\mathcal{F}_{i}^{c}$ is a polynomial. Using Assumption 5 we obtain the averaged equation with error term

$$
a_{i}(t)=a_{i}(0)+\int_{0}^{t} \overline{\mathcal{F}}_{i}(a) d \tau+\tilde{R}(t)
$$

where

$$
\tilde{R}(t)=R_{1}(t)+\mathcal{O}\left(\varepsilon^{1-m \kappa}\right) .
$$

To bound $\tilde{R}$ we use Lemmas 13 and 11 and the fact that $\mathcal{F}_{i}$ is a polynomial.

Let us now summarize all the error estimates in a single set in probability space.

Definition 15 Define the set $\Omega^{*} \subset \Omega$ such that all of the following estimates hold on $\Omega^{*}$

$$
\sup _{\left[0, \tau^{*}\right]}\|\psi-\mathcal{Q}\|_{\alpha}<C \varepsilon^{2-m \kappa-\kappa}
$$




$$
\begin{gathered}
\sup _{\left[0, \tau^{*}\right]}\|\psi\|_{\alpha}<C \varepsilon^{-\frac{3}{2} \kappa_{0}}, \\
\sup _{\left[0, \tau^{*}\right]}\|\tilde{R}\|_{\alpha}<C \varepsilon^{1-2 m \kappa-\kappa},
\end{gathered}
$$

and

$$
\sup _{\left[0, T_{1}\right]}\|b\|_{\alpha} \leq C_{0}
$$

The following lemmas show that the set $\Omega^{*}$ has probability close to 1 provided $b$ is not too large.

Proposition 16 Under Assumptions 3 and 5 the set $\Omega^{*}$ has probability

$$
\mathbb{P}\left(\Omega^{*}\right) \geq 1-C \varepsilon^{p}-\mathbb{P}\left(\sup _{\left[0, T_{1}\right]}\|b\|_{\alpha}>C_{0}\right) .
$$

Proof. First

$$
\begin{gathered}
\mathbb{P}\left(\Omega^{*}\right) \geq 1-\mathbb{P}\left(\sup _{\left[0, \tau^{*}\right]}\|\psi-\mathcal{Q}\|_{\alpha} \geq C \varepsilon^{-3 \kappa}\right)-\mathbb{P}\left(\sup _{\left[0, \tau^{*}\right]}\|\psi\|_{\alpha} \geq C \varepsilon^{-\frac{3}{2} \kappa_{0}}\right) \\
-\mathbb{P}\left(\sup _{\left[0, \tau^{*}\right]}\|\tilde{R}\|_{\alpha} \geq C \varepsilon^{1-2 m \kappa-\kappa}\right)-\mathbb{P}\left(\sup _{\left[0, T_{1}\right]}\|b\|_{\alpha}>C_{0}\right) .
\end{gathered}
$$

Using Chebychev inequality and Lemmas 10, 11 and 12, we obtain for $\kappa>\kappa_{0}$ and sufficiently large $q>\frac{2 p}{\left(\kappa-\kappa_{0}\right)}>0$

$$
\begin{aligned}
\mathbb{P}\left(\Omega^{*}\right) & \geq 1-C\left[\varepsilon^{q \kappa}+\varepsilon^{\frac{1}{2} q \kappa}+\varepsilon^{q\left(\kappa-\kappa_{0}\right)}\right]-\mathbb{P}\left(\sup _{\left[0, T_{1}\right]}\|b\|_{\alpha}>C_{0}\right) \\
& \geq 1-C \varepsilon^{p}-\mathbb{P}\left(\sup _{\left[0, T_{1}\right]}\|b\|_{\alpha}>C_{0}\right) .
\end{aligned}
$$

Theorem 17 Assume that Assumptions 3 and 5 hold. Suppose $a(0)=\mathcal{O}(1)$ and $\psi(0)=\mathcal{O}(1)$. Let $b$ be a solution of (2) and a as defined in (22). If the initial conditions satisfy $a(0)=b(0)$, then for $\kappa<\frac{1}{2 m+1}$ we obtain

$$
\sup _{t \in\left[0, T_{1}\right]}\|a(t)-b(t)\|_{\alpha} \leq C \varepsilon^{1-2 m \kappa-\kappa} \quad \text { on } \Omega^{*},
$$

and

$$
\sup _{t \in\left[0, T_{1}\right]}\|a(t)\|_{\alpha} \leq C \quad \text { on } \Omega^{*} .
$$

Proof. Subtracting (2) from (22) and defining

$$
h(t):=a(T)-b(t),
$$


we obtain

$$
h(t)=\int_{0}^{t}\left[\overline{\mathcal{F}}^{c}(h+b)-\overline{\mathcal{F}}^{c}(b)\right] d \tau+\tilde{R}(t),
$$

where the error $\tilde{R}$ is bounded by

$$
\tilde{R}=\mathcal{O}\left(\varepsilon^{1-2 m \kappa}\right) .
$$

Define $Q$ as

$$
Q(t):=h(t)-\tilde{R}(t)
$$

From (34) we obtain

$$
\partial_{t} Q=\overline{\mathcal{F}}^{c}(Q+\tilde{R}+b)-\overline{\mathcal{F}}^{c}(b) .
$$

Taking the scalar product $\langle Q, \cdot\rangle$ in $\mathcal{H}^{\alpha}$ on both sides, yields

$$
\frac{1}{2} \partial_{t}\|Q\|_{\alpha}^{2}=\left\langle\overline{\mathcal{F}}^{c}(Q+\tilde{R}+b)-\overline{\mathcal{F}}^{c}(b), Q\right\rangle_{\mathcal{H}^{\alpha}} .
$$

Using Young and Cauchy-Schwartz inequalities, where $\overline{\mathcal{F}}^{c}$ is a polynomial of degree $m$, we obtain

$$
\frac{1}{2} \partial_{t}\|Q\|_{\alpha}^{2} \leq C\left(1+\|Q\|_{\alpha}^{m-1}+\|\tilde{R}\|_{\alpha}^{m-1}+\|b\|_{\alpha}^{m-1}\right)\left(\|Q\|_{\alpha}^{2}+\|\tilde{R}\|_{\alpha}^{2}\right) .
$$

As long as $\|Q(t)\|<1$, using Equations (28) and (29), we obtain for $\kappa<$ $\frac{1}{2 m+1}$

$$
\frac{1}{2} \partial_{t}\|Q(t)\|_{\alpha}^{2} \leq c\|Q(t)\|_{\alpha}^{2}+C \varepsilon^{2-2(2 m+1) \kappa} \text { on } \Omega^{*} .
$$

Using Gronwall's lemma, we obtain for $t \leq \tau^{*} \wedge T_{1} \leq T_{0}$

$$
\|Q(t)\|_{\alpha} \leq C \varepsilon^{1-(2 m+1) \kappa} e^{2 c T_{0}}
$$

and thus $\|Q(t)\|_{\alpha}<1$ for $t \leq \tau^{*} \wedge T_{1}$. Taking supremum on $\left[0, \tau^{*} \wedge T_{1}\right]$

$$
\sup _{t \in\left[0, \tau^{*} \wedge T_{1}\right]}\|Q(t)\|_{\alpha} \leq C \varepsilon^{1-(2 m+1) \kappa} \text { on } \Omega^{*} .
$$

Hence,

$$
\begin{aligned}
\sup _{\left[0, \tau^{*} \wedge T_{1}\right]}\|a-b\|_{\alpha} & =\sup _{\left[0, \tau^{*} \wedge T_{1}\right]}\|Q-\tilde{R}\|_{\alpha} \leq \sup _{\left[0, \tau^{*} \wedge T_{1}\right]}\|Q\|_{\alpha}+\sup _{\left[0, \tau^{*} \wedge T_{1}\right]}\|\tilde{R}\|_{\alpha} \\
& \leq C \varepsilon^{1-(2 m+1) \kappa} \text { on } \Omega^{*} .
\end{aligned}
$$

We finish the proof by using (33), (36) and

$$
\sup _{\left[0, \tau^{*} \wedge T_{1}\right]}\|a\|_{\alpha} \leq \sup _{\left[0, \tau^{*} \wedge T_{1}\right]}\|a-b\|_{\alpha}+\sup _{\left[0, \tau^{*} \wedge T_{1}\right]}\|b\|_{\alpha} \leq C .
$$


This implies $T_{1} \leq \tau^{*}$ on $\Omega^{*}$. Thus, we have on $\Omega^{*}$

$$
\sup _{\left[0, T_{1}\right]}\|a-b\|_{\alpha} \leq C \varepsilon^{1-(2 m+1) \kappa},
$$

and

$$
\sup _{\left[0, T_{1}\right]}\|a\|_{\alpha} \leq C
$$

Now, we can use the results obtained above to prove the main result from Theorem 9 for the system of SPDE (1).

Proof of Theorem 9. For the stopping time we note that (from the previous proof)

$$
\Omega \supset\left\{\tau^{*}>T_{1}\right\} \supseteq\left\{\sup _{\left[0, \tau^{*} \wedge T_{1}\right]}\|a\|_{\alpha}<\varepsilon^{-\kappa}, \sup _{\left[0, \tau^{*} \wedge T_{1}\right]}\|\psi\|_{\alpha}<\varepsilon^{-\kappa}\right\} \supseteq \Omega^{*} .
$$

Now let us turn to the approximation result. Using (11) and triangle inequality, we obtain

$$
\begin{aligned}
\sup _{t \in\left[0, T_{1}\right]}\|u(t)-b(t)-\mathcal{Q}(t)\|_{\alpha} & \leq \sup _{\left[0, T_{1}\right]}\|a-b\|_{\alpha}+\sup _{\left[0, T_{1}\right]}\|\psi-\mathcal{Q}\|_{\alpha} \\
& \leq \sup _{\left[0, T_{1}\right]}\|a-b\|_{\alpha}+\sup _{\left[0, \tau^{*}\right]}\|\psi-\mathcal{Q}\|_{\alpha} .
\end{aligned}
$$

From (26) and (31), we obtain

$$
\sup _{t \in\left[0, T_{1}\right]}\|u(t)-b(t)-\mathcal{Q}(t)\|_{\alpha} \leq C \varepsilon^{1-(2 m+1) \kappa} \text { on } \Omega^{*} .
$$

Hence,

$$
\mathbb{P}\left(\sup _{t \in\left[0, T_{1}\right]}\|u(t)-b(t)-\mathcal{Q}(t)\|_{\alpha}>C \varepsilon^{1-(2 m+1) \kappa}\right) \leq 1-\mathbb{P}\left(\Omega^{*}\right) .
$$

Using (30), yields (16).

\section{$5 \quad$ Averaging}

In this section, we discuss how the part on averaging in Assumption 5 can be verified in the setting of a quite general example. Note that here we always assume that the Brownian motions in the Wiener-process are independent.

In the first lemma we state the averaging Lemma 5.1 over the fast OUprocess from our paper [4]. Actually, this is a slightly generalized version, which we state without proof. The key idea is a straightforward calculation based on Itô's formula applied to $d\left(X Z^{\ell}\right)$ together with the formulas for $d X$ and $d Z_{i}$. 
Lemma 18 Let $X$ be a real valued stochastic process such that for some $r \geq 0$ we have $X(0)=\mathcal{O}\left(\varepsilon^{-r}\right)$. Suppose $Z=\left(Z_{1}, \ldots ., Z_{n}\right)$ with

$$
Z_{i}(t)=\hat{\rho}_{i} \varepsilon^{-1} \int_{0}^{t} e^{-\varepsilon^{-2}(t-s) \hat{\lambda}_{i}} d B_{i}(s),
$$

for some $\hat{\rho}_{i}, \hat{\lambda}_{i} \in \mathbb{R}$ and independent Brownian motion $B_{i}$, are fast $O U$ process. Fix small $\kappa_{0}>0$. If $d X=G d T$ with $G=\mathcal{O}\left(\varepsilon^{-r}\right)$, then for $\ell \in \mathbb{N}_{0}^{n}$

$$
\int_{0}^{t} X Z^{\ell} d \tau=\sum_{i=1}^{n} \frac{\ell_{i}\left(\ell_{i}-1\right) \hat{\rho}_{i}^{2}}{2 \sum_{j=1}^{n} \ell_{j} \hat{\lambda}_{j}} \int_{0}^{t} X Z^{\ell} Z_{i}^{-2} d \tau+\mathcal{O}\left(\varepsilon^{1-r-\kappa_{0}}\right) .
$$

In the next lemma we apply the previous Lemma 18 iteratively, and verify a result which we need later to determine the averaged nonlinearity $\overline{\mathcal{F}}_{i}$.

Lemma 19 Let $X$ be as in Lemma 18 and consider $\mathcal{Z}$ from definition 4 under assumption 3, where we additionally suppose that all $\beta_{i, k}$ are independent.

Then, for $\ell \in \mathbb{N}_{0}^{n}$ and $|\ell| \geq 1$, we obtain

1. If one of the $\ell_{i}$ is odd, then

$$
P_{c} \int_{0}^{t} X \mathcal{Z}^{\ell} d \tau=\mathcal{O}\left(\varepsilon^{1-r-\kappa_{0}}\right)
$$

2. If all $\ell_{i}$ are even, then there is a constant $C_{\ell}$ such that

$$
P_{c} \int_{0}^{t} X \mathcal{Z}^{\ell} d \tau=C_{\ell} \int_{0}^{t} X d \tau+\mathcal{O}\left(\varepsilon^{1-r-\kappa_{0}}\right) .
$$

Proof. We proceed by looking at three cases. The first one is forcing only on a single mode, while the second case treats the case of $N=2$, and finally we comment briefly on the general case.

First case: Assume that for some $k \in \mathbb{N}^{n}$ we have

$$
\mathcal{Z}_{i}(t)=\mathcal{Z}_{i k_{i}} e_{k_{i}}=\rho_{i, k_{i}} \varepsilon^{-1} \int_{0}^{t} e^{-\varepsilon^{-2}(t-\tau) d_{i} \lambda_{k_{i}}} d \beta_{k_{i}}(\tau) e_{k_{i}} .
$$

Thus (recall that $X$ is real valued)

$$
P_{c} \int_{0}^{t} X \mathcal{Z}^{\ell} d \tau=\int_{0}^{t} X \prod_{i=1}^{n} \mathcal{Z}_{i k_{i}}^{\ell_{i}} d \tau \cdot P_{c} \prod_{i=1}^{n} e_{k_{i}}^{\ell_{i}} .
$$

If one of the $\ell_{i}$ is odd, then applying Lemma 18 inductively yields

$$
P_{c} \int_{0}^{t} X \mathcal{Z}^{\ell} d \tau=\mathcal{O}\left(\varepsilon^{1-r-\kappa_{0}}\right)
$$


If all $\ell_{i}$ are even, we obtain by Lemma 18

$P_{c} \int_{0}^{t} X \mathcal{Z}^{\ell} d \tau=\sum_{i=1}^{n} \frac{\ell_{i}\left(\ell_{i}-1\right) \rho_{i, k_{i}}^{2}}{2 \sum_{j=1}^{n} \ell_{j} d_{j} \lambda_{k_{j}}} \int_{0}^{t} X P_{c}\left(\prod_{i=1}^{n} e_{k_{i}}^{\ell_{i}}\right) \prod_{j=1}^{n} \mathcal{Z}_{j, k_{j}}^{\ell_{j}} \mathcal{Z}_{i, k_{i}}^{-2} d \tau+\mathcal{O}\left(\varepsilon^{1-r-\kappa_{0}}\right)$.

Proceeding inductively, we obtain the existence of the constant $C_{\ell}$, once all $\mathcal{Z}$ 's are eliminated.

Second case: Here we assume $N=2$. Thus

$$
\mathcal{Z}_{i}=\mathcal{Z}_{i 1} e_{1}+\mathcal{Z}_{i 2} e_{2}
$$

Using binomial formula $(x+y)^{\ell}=\sum_{\substack{r \in \mathbb{N}_{0}^{n} \\ r \leq \ell}}\left(\begin{array}{c}\ell_{i} \\ r\end{array}\right) x^{r} y^{\ell-r}$ for $\ell \in \mathbb{N}_{0}^{n}$, and $x, y \in \mathbb{R}$. We obtain

$$
P_{c} \int_{0}^{t} X \mathcal{Z}^{\ell} d \tau=\sum_{\substack{r \in \mathbb{N}_{0}^{n} \\
r \leq \ell}} \int_{0}^{t} X P_{c}\left(\left(\begin{array}{l}
\ell \\
r
\end{array}\right) e_{1}^{|r|} e_{2}^{|\ell-r|}\right) \mathcal{Z}_{\bullet, 1}^{r} \mathcal{Z}_{\bullet, 2}^{\ell-r} d \tau
$$

where $\mathcal{Z}_{\bullet, 1}=\left(\mathcal{Z}_{1,1}, \ldots \ldots \ldots, \mathcal{Z}_{n, 1}\right)$. Thus using Lemma 18 we can proceed as in the first case.

Third case: For $N>2$, we can proceed similar to the second case by expanding

$$
\left(\sum_{j=1}^{N} \mathcal{Z}_{i j} e_{j}\right)^{\ell}
$$

Now, let us give an explicit formula for the polynomial $\overline{\mathcal{F}}_{i}(b)$ which is defined in (2) and Assumption 5. Usually, one would use integration with respect to the invariant measure of the OU-process to determine $\overline{\mathcal{F}}_{i}$. Here, we give a simple proof for a particular example, which is needed later.

Corollary 20 Under the assumptions of Lemma 19 consider the case of single mode forcing as given in Equation (40). Let $\mathcal{F}_{i}$ be a polynomial of degree $m_{i}$, then the averaging part of Assumption 5 is true with

$$
\overline{\mathcal{F}}_{i}(b)=\mathcal{F}_{i}(b)+\sum_{|\ell| \geq 1} \frac{C_{2 \ell}}{(2 \ell) !} D^{2 \ell} \mathcal{F}_{i}(b)
$$

where

$$
C_{2 \ell}=\sum_{i=1}^{n} \frac{\ell_{i}\left(2 \ell_{i}-1\right) \rho_{i, k_{i}}^{2}}{2 \sum_{j=1}^{n} \ell_{j} d_{j} \lambda_{k_{j}}} P_{c}\left(\prod_{j=1}^{n} e_{k_{j}}^{2 \ell_{j}}\right) C_{2 \ell-2 f_{i}},
$$

where $f_{i}=(0, \ldots, \underbrace{1}_{i-t h}, \ldots, 0)$ is the standard basis in $\mathbb{R}^{n}$ and $C_{0}=1$. 
Proof. Applying Taylor's expansion for the function $\mathcal{F}_{i}^{c}: \mathcal{H} \rightarrow \mathbb{R}$, yields

$$
\int_{0}^{t} \mathcal{F}_{i}^{c}(b+\mathcal{Z}) d \tau=\sum_{|\ell| \geq 0} P_{c} \int_{0}^{t} \frac{D^{\ell} \mathcal{F}_{i}(b)}{\ell !} \mathcal{Z}^{\ell} d \tau .
$$

Applying Lemma 19 to Equation (43), yields

$$
\int_{0}^{t} \mathcal{F}_{i}^{c}(b+\mathcal{Z}) d \tau=\sum_{|\ell| \geq 0} \frac{C_{\ell}}{\ell !} \int_{0}^{t} D^{\ell} \mathcal{F}_{i}(b) d \tau+\mathcal{O}\left(\varepsilon^{1-m_{i} \kappa}\right),
$$

where $C_{0}=1$ and $C_{\ell}=0$ if one $\ell_{i}$ is odd. Thus

$$
\int_{0}^{t} \mathcal{F}_{i}^{c}(b+\mathcal{Z}) d \tau=\sum_{|\ell| \geq 0} \frac{C_{2 \ell}}{(2 \ell) !} \int_{0}^{t} D^{2 \ell} \mathcal{F}_{i}(b) d \tau+\mathcal{O}\left(\varepsilon^{1-m_{i} \kappa}\right) .
$$

From the first case of the proof of Lemma 19, we obtain (42).

Example 21 Let the noise force only one single mode, assume $n=2$ and $d=1$, such that the eigenfunction are given by $e_{0}=1$ and $e_{k}=\sqrt{2} \cos (\pi k x)$ for $k>0$. We consider two different cases.

First case: If $|\ell|=1$, then $\left(\ell_{1}, \ell_{2}\right)=(1,0)$ or $(0,1)$ hence

$$
C_{(2,0)}=\frac{\rho_{1, k_{1}}^{2}}{2 d_{1} \lambda_{k_{1}}}\left(P_{c} e_{k_{1}}^{2}\right) C_{(0,0)}=\frac{\rho_{1, k_{1}}^{2}}{2 d_{1} \lambda_{k_{1}}} .
$$

and

$$
C_{(0,2)}=\frac{\rho_{2, k_{2}}^{2}}{2 d_{2} \lambda_{k_{2}}} .
$$

Second case: If $|\ell|=2$, then $\left(\ell_{1}, \ell_{2}\right)=(2,0),(1,1)$ or $(0,2)$ hence

$$
\begin{aligned}
& C_{(4,0)}=\frac{9 \rho_{1, k_{1}}^{2}}{4 d_{1} \pi^{2} k_{1}^{2}} C_{(2,0)}=\frac{9 \rho_{1, k_{1}}^{4}}{8 d_{1}^{2} \pi^{4} k_{1}^{4}}, \\
& C_{(0,4)}=\frac{9 \rho_{2, k_{2}}^{2}}{4 d_{2} \pi^{2} k_{2}^{2}} C_{(2,0)}=\frac{9 \rho_{2, k_{2}}^{4}}{8 d_{2}^{2} \pi^{4} k_{2}^{4}},
\end{aligned}
$$

and

$$
\begin{aligned}
C_{(2,2)} & =\frac{1}{2\left(d_{1} \lambda_{k_{1}}+d_{2} \lambda_{k_{2}}\right)} P_{c}\left(e_{k_{1}}^{2} e_{k_{2}}^{2}\right)\left[\rho_{1, k_{1}}^{2} C_{(0,2)}+\rho_{2, k_{2}}^{2} C_{(2,0)}\right] \\
& =\frac{\rho_{1, k_{1}}^{2} \rho_{2, k_{2}}^{2}}{2\left(d_{1} \lambda_{k_{1}}+d_{2} \lambda_{k_{2}}\right)} P_{c}\left(e_{k_{1}}^{2} e_{k_{2}}^{2}\right),
\end{aligned}
$$

where we used (45) and (46). Now, we have two different cases, first assume $k_{1} \neq k_{2}$. In this case $P_{c}\left(e_{k_{1}}^{2} e_{k_{2}}^{2}\right)=1$, and

$$
C_{(2,2)}=\frac{\rho_{1, k_{1}}^{2} \rho_{2, k_{2}}^{2}}{2\left(d_{1} \lambda_{k_{1}}+d_{2} \lambda_{k_{2}}\right)} .
$$


For the second case consider $k_{1}=k_{2}$. In this case $P_{c}\left(e_{k_{1}}^{2} e_{k_{2}}^{2}\right)=\frac{3}{2}$, and

$$
C_{(2,2)}=\frac{3 \rho_{1, k_{1}}^{2} \rho_{2, k_{2}}^{2}}{4\left(d_{1} \lambda_{k_{1}}+d_{2} \lambda_{k_{2}}\right)}
$$

\section{Examples}

In this section we consider simple examples with homogeneous Neumann boundary condition on the interval $[0,1]$, in order to illustrate our results.

Let us first define the linear operator that fits into the setting of Definition 1. Suppose the eigenfunctions of the operator $-\mathcal{A}_{i}=-d_{i} \partial_{x}^{2}$ are

$$
e_{k}=\left\{\begin{array}{lr}
1 & \text { if } k=0 \\
\sqrt{2} \cos (\pi k x) & \text { if } k>0
\end{array}\right.
$$

and the kernel

$$
\mathcal{N}=\operatorname{span}\{1\} .
$$

The corresponding eigenvalues are $\lambda_{k}=\pi^{2} k^{2}$.

In order to have different nonlinearities, we consider two applications. One is motivated from biology and the other one from chemistry.

\subsection{Biological Application}

We consider a simple predator-prey system with diffusion which is a modified Lotka-Volterra system with logistic growth of the prey and with both predator and prey dispersing by diffusion (cf. J. Murray [24]). Also, Dunbar $[13,14]$ discussed this model in detail. We consider a slightly unphysical model in the sense that $u_{i}<0$ is possible in the model. Thus its validity is restricted until times where $u_{i}$ reaches 0 .

The model with additive noise takes the form

$$
\begin{aligned}
\partial_{t} u_{1} & =\frac{d_{1}}{\varepsilon^{2}} \frac{\partial^{2} u_{1}}{\partial x^{2}}+A u_{1}\left(1-\frac{u_{1}}{K}\right)-B u_{1} u_{2}+\varepsilon^{-1} \partial_{t} W_{1}(t), \\
\partial_{t} u_{2} & =\frac{d_{2}}{\varepsilon^{2}} \frac{\partial^{2} u_{2}}{\partial x^{2}}+C u_{1} u_{2}-D u_{2}+\varepsilon^{-1} \partial_{t} W_{2}(t),
\end{aligned}
$$

where $u_{1}$ is the prey, $u_{2}$ is the predator, $A, B, C, D$ and $K$, the prey carrying capacity, are positive constants. We assume very fast diffusion $\frac{d_{i}}{\varepsilon^{2}}$, and in addition large fluctuation of order $\varepsilon^{-1}$. If we take

$$
W_{1}(t)=\rho_{1} \beta_{1}(t) \cos (\pi x) \text { and } W_{2}(t)=\rho_{2} \beta_{2}(t) \cos (\pi x),
$$

then the noise is large, but it does not change the average population size. This is perfectly in the setting of Assumption 3 and Corollary 20 and thus Assumption 5 is true, and we can read off $\overline{\mathcal{F}}_{i}$ from example 21. 
Our main theorem states that with high probability

$$
u(t)=b(t)+\mathcal{Z}(t)+\mathcal{O}\left(\varepsilon^{1-}\right),
$$

on finite time horizons, as long as the approximation $b$ remains bounded. Here

$$
u=\left(\begin{array}{l}
u_{1} \\
u_{2}
\end{array}\right), \quad b=\left(\begin{array}{l}
b_{1} \\
b_{2}
\end{array}\right), \text { and } \mathcal{Z}=\left(\begin{array}{l}
\mathcal{Z}_{1} \\
\mathcal{Z}_{2}
\end{array}\right)
$$

where $b_{1}$ and $b_{2}$ are the solutions of

$$
\begin{aligned}
& \partial_{t} b_{1}=A b_{1}\left(1-\frac{b_{1}}{K}\right)-B b_{1} b_{2}-\frac{A \rho_{1}^{2}}{2 d_{1} \pi^{2} K}, \\
& \partial_{t} b_{2}=C b_{1} b_{2}-D b_{2},
\end{aligned}
$$

and

$$
\mathcal{Z}_{i}=\varepsilon^{-1} \rho_{i} \int_{0}^{t} e^{-\varepsilon^{-2}(t-s) d_{1} \pi^{2}} d \beta_{i}(t) \cos (\pi x) \text { for } i=1,2 .
$$

The biological effect of the $\frac{A \rho_{1}^{2}}{2 d_{1} \pi^{2} K}$ term can be understood by neglecting $b_{2}$, i.e assuming no predators. In this case one gets

$$
\partial_{t} b_{1}=A b_{1}\left(1-\frac{b_{1}}{K}\right)-\frac{A \rho_{1}^{2}}{2 d_{1} \pi^{2} K} .
$$

There appears a noise-induced effective extra drift term from the combination of fast diffusion and large fluctuations, although both terms individually have no direct impact on the average population size. If

$$
b_{1}<\frac{\rho_{1}^{2}}{2 d_{1} \pi^{2} K},
$$

then $\frac{d b_{1}}{d t}<0$, i.e. it decreases. Since the effect of $b_{2}$ is to further decrease $b_{1}$ then Equation (47) implies the annihilation of the system, as $b_{1}$ will cross 0 , which means that the prey dies out, and the model is unphysical afterwards.

\subsection{Chemical Application}

Let us consider a very similar model to the previous one, only with different nonlinearities. Therefore, we will discuss this very briefly only. A simple archetype example for a reaction-diffusion system is a cubic auto-catalytic reaction between two chemicals according to the rule $A+B \rightarrow 2 B$ with rate $r=\eta u_{1} u_{2}^{2}$ (cf. [29]).

Denoting by $u_{1}$ and $u_{2}$ the concentration of $A$ and $B$, respectively. The two species satisfy the equations:

$$
\begin{aligned}
& \partial_{t} u_{1}=\frac{d_{1}}{\varepsilon^{2}} \frac{\partial^{2} u_{1}}{\partial x^{2}}-\eta u_{1} u_{2}^{2}+\varepsilon^{-1} \partial_{t} W_{1}(t), \\
& \partial_{t} u_{2}=\frac{d_{2}}{\varepsilon^{2}} \frac{\partial^{2} u_{2}}{\partial x^{2}}+\eta u_{1} u_{2}^{2}+\varepsilon^{-1} \partial_{t} W_{2}(t),
\end{aligned}
$$


We consider again large diffusion and large noise which both might be introduced by fast stirring.

If we take

$$
W_{1}(t)=\rho_{1} \beta_{1}(t) \cos (\pi x) \quad \text { and } \quad W_{2}(t)=\rho_{2} \beta_{2}(t) \cos (\pi x),
$$

then our main theorem states that

$$
u(t)=b(t)+\mathcal{Z}(t)+\mathcal{O}\left(\varepsilon^{1-}\right),
$$

with

$$
u=\left(\begin{array}{l}
u_{1} \\
u_{2}
\end{array}\right), \quad b=\left(\begin{array}{c}
b_{1} \\
b_{2}
\end{array}\right), \text { and } \mathcal{Z}=\left(\begin{array}{c}
\mathcal{Z}_{1} \\
\mathcal{Z}_{2}
\end{array}\right)
$$

where $b_{1}$ and $b_{2}$ are the solutions of

$$
\begin{aligned}
\partial_{t} b_{1} & =-\eta b_{1} b_{2}^{2}-\frac{\eta \rho_{2}^{2}}{2 d_{2} \pi^{2}} b_{1}, \\
\partial_{t} b_{2} & =\eta b_{1} b_{2}^{2}+\frac{\eta \rho_{2}^{2}}{2 d_{2} \pi^{2}} b_{1} .
\end{aligned}
$$

We note that high fluctuations in combination with fast diffusion lead to effective new terms describing the transformation of $b_{1}$ to $b_{2}$. Again both terms individually do not change the average $\int u_{i} d x=b_{i}$, only their nonlinear combination does.

Let us check the bound on $b$. We note that $\sum_{i=1}^{n} \partial_{t} b_{i}=0$, and thus integrating from 0 to $t$ yields

$$
\sum_{i=1}^{n} b_{i}(t)=\sum_{i=1}^{n} b_{i}(0)=C_{0} .
$$

As $b_{i} \geq 0$, we have

$$
0 \leq b_{i}(t) \leq \sum_{i=1}^{n} b_{i}(t) \leq C_{0} .
$$

Hence, we obtain for all times $t>0$

$$
\|b(t)\|=\left(\sum_{i=1}^{2} b_{i}^{2}(t)\right)^{1 / 2} \leq C_{0} \sqrt{2} .
$$

Thus the approximation in this case is valid on any fixed finite time interval.

\section{Acknowledgements}

This work was supported by the Deutsche Forschungsgemeinschaft (DFG) 'Multiscale Analysis of SPDEs' (DFG BL535/9-2). 


\section{References}

[1] D. Blömker. Amplitude equations for locally cubic non-autonomous nonlinearities, SIAM Journal on Applied Dynamical Systems, 2:464$486,(2003)$.

[2] D. Blömker. Non-homogeneous noise and Q-Wiener processes on bounded domains. Stochastic Analysis and Applications, 23(2):255-273, 2005.

[3] D. Blömker and W. W. Mohammed. Amplitude equations for SPDEs with quadratic nonlinearities. Electronic Journal of Probability, 14:2527-2550, (2009).

[4] D. Blömker and W. W. Mohammed. Amplitude equations for SPDEs with cubic nonlinearities. Stochastics An International Journal of Probability and Stochastic Process, 85:181-215 (2013).

[5] D. Blömker, M. Hairer, and G.A. Pavliotis. Multiscale analysis for stochastic partial differential equations with quadratic nonlinearities. Nonlinearity, 20:1-25 (2007).

[6] A. Bruckner and Y. K. Lin, Application of complex stochastic averaging to non-linear random vibration problems. Int. J. Non-linear Mech. 22:237-250 (1987).

[7] S. Cerrai. A Khasminskii type averaging principle for stochastic reaction-diffusion equations, Annals of Applied Probability, 19:899-948 (2009).

[8] S. Cerrai. Normal deviations from the averaged motion for some reaction-diffusion equations with fast oscillating perturbation, Journal des Mathematiques Pures et Appliquees, 91:614-647(2009).

[9] S. Cerrai. Averaging principle for systems of RDEs with polynomial nonlinearities perturbed by multiplicative noise, SIAM Journal of Mathematical Analysis, 43:2482-2518(2011).

[10] S. Cerrai and M. Freidlin. Averaging principle for a class of SPDE's, Probability Theory and Related Fields, 144:137-177 (2009).

[11] R. Courant and D. Hilbert. Methoden der mathematischen Physik. (Methods of mathematical physics). 4. Aufl. (German) Springer-Verlag, (1993).

[12] G. Da Prato, J. Zabczyk. Stochastic equations in infinite dimensions. Vol. 44 of Encyclopedia of Mathematics and its Applications. Cambridge University Press, Cambridge, (1992). 
[13] S. R. Dunbar. Traveling wave solutions of diffusive Lotka-Volterra equations. J. Math. Biol., 17:11-32, (1983).

[14] S. R. Dunbar. Traveling wave solutions of diffusive Lotka-Volterra equations: A heteroclinic connection in $R^{4}$. Trans. Amer. Math. Soc., 268:557-594 (1984).

[15] W. Ebeling, H. Herzel, W. Richert and L. Schimansky-Geier. Influence of noise on Duffing-van der Pol oscillators. Zeitschrift für Angewandte Mathematik und Mechanik, 66:141-146, (1986).

[16] F. Flandoli. A stochastic reaction-diffusion equation with multiplicative noise. Appl. Math. Lett., 4:45-48, (1991).

[17] D. Henry. Geometric Theory of Semilinear Parabolic Equations, Lecture Notes in Mathematics 840, Springer-Verlag, Berlin, (1981).

[18] A. Hutt. Additive noise may change the stability of nonlinear systems, Europhys. Lett., 84(3):34003, (2008).

[19] A. Hutt, A. Longtin, and L. Schimansky-Geier, Additive noise-induced Turing transitions in spatial systems with application to neural fields and the Swift-Hohenberg equation, Physica D, 237:755-773, (2008).

[20] K. Klepel, D. Blömker, W. W. Mohammed. Amplitude equation for the generalized Swift Hohenberg equation with noise ZAMP - Zeitschrift für angewandte Mathematik und Physik, 65(6):1107-1126, 2013.

[21] T. G. Kurtz. A limit theorem for perturbed operator semigroups with applications to random evolutions. Journal of functional analysis, 2:55$67,(1973)$.

[22] A. Majda, I. Timofeyev, E. Vanden-Eijnden. Stochastic models for selected slow variables in large deterministic systems. Nonlinearity, 19:769-794 (2006)

[23] W. W. Mohammed, D. Blömker, K. Klepel, Multi-scale analysis of SPDEs with degenerate additive noise. Journal of Evolution Equations 14(2):273-298, (2014).

[24] J. Murray. Mathematical Biology, II: Spatial Models and Biomedical Applications. Springer, (2003).

[25] A.J. Roberts. A step towards holistic discretisation of stochastic partial differential equations. ANZIAM J., 45(E):C1-C15, (2003).

[26] T. Runst and W. Sickel. Sobolev spaces of fractional order, Nemytskij operators, and nonlinear partial differential equations. Walter de Gruyter. Berlin. New York, (1996). 
[27] G. Pavliotis and A. Stuart. Multiscale Methods Averaging and Homogenization. Springer, Berlin Heidelberg New York, (2007).

[28] A. Pazy. Semigroups of linear operators and applications to partial differential equations. Applied Mathematical Sciences, 44. New York etc.: Springer-Verlag, Berlin (1983).

[29] A. H. Salas, L. J. Martinez and S. O. Fernandez. Reaction-diffusion equations: A chemical application. Scientia Et Technica. XVII (46):134-137,(2010). 